\title{
Chemical and microbiological characterization of primary biological aerosol particles at the boreal forest
}

Jose Ruiz-Jimenez ${ }^{1,5}$, Magdalena Okuljar ${ }^{1,5}$, Outi-Maaria Sietiö ${ }^{2,4}$, Giorgia Demaria ${ }^{1}$, Thanaporn Liangsupree $^{1}$, Elisa Zagatti ${ }^{1}$, Juho Aalto ${ }^{4}$, Kari Hartonen ${ }^{1,5}$, Jussi Heinonsalo ${ }^{3}$, Jaana Bäck ${ }^{4}$, Tuukka Petäjä $^{5}$ and Marja-Liisa Riekkola ${ }^{1,5}$

\footnotetext{
${ }^{1}$ Department of Chemistry, P.O. Box 55, FI-00014 University of Helsinki, Finland

${ }^{2}$ Department of Microbiology, P.O. Box 56, FI-00014University of Helsinki, Finland

${ }^{3}$ Department of Forest Sciences, P.O. Box 27, FI-00014 University of Helsinki, Finland

${ }^{4}$ Institute for Atmospheric and Earth System Research/Forest Sciences, Faculty of Agriculture and Forestry, P.O. Box 64, FI-00014 University of Helsinki, Finland

${ }^{5}$ Institute for Atmospheric and Earth System Research, Faculty of Science, P.O. Box 64, FI-00014 University of Helsinki, Finland
}

Correspondence to: Marja-Liisa Riekkola (marja-liisa.riekkola@helsinki.fi)

S1. Material and reagents.

S2. Determination of gas phase volatile organic compounds.

Table S1. Aerosol sampling dates and sampling volume.

Table S2. Ionization conditions for amino acids and saccharides.

Table S3. Multiple reaction monitoring settings for amino acids and saccharides.

Table S4. Meteorological and environmental parameters selected for statistical analysis.

Table S5. Analytical features of the method used for the chemical characterization of the PBAPs.

Table S6 Extraction recoveries of the method used for the chemical characterization of the PBAPs.

Table S7. Effect of the sample matrix on the ionization of the different amino acids and saccharides analyzed by the method used for the chemical characterization of the PBAPs.

Table S8. Summary statistics for the determination of amino acids in the PBAPs.

Table S9. Summary statistics for the determination of saccharides in PBAPs. 
Table S10. Summary statistics for the determination of number of gene copies for microbes in PBAPs. Table S11. Summary statistics for the determination of total DNA concentration in PBAPs.

Table S12. Membership table for the cluster classification of the samples based on variations in meteorological conditions.

Table S13. Statistical features of the MLR models used for the evaluation of the chemical composition influencing the variation of the microbial groups in the aerosol particles.

Table S14. Statistical features of the MLR models used for the evaluation of the gas phase VOCs influencing the variation of the microbial groups in PBAPs.

Figure S1 Typical chromatograms provided by standard solutions and aerosol samples.

Figure S2. Pearson correlation for the individual microbial groups and chemical compounds detected in PBAPs as a function of the particle size.

Figure S3. Differences between clusters based on the meteorological and environmental variables.

Figure S4. Pearson correlation results between the chemical and the microbial groups for the different particle sizes.

Figure S5. VOCs concentration in the gas phase.

Figure S6. Influence of the gas phase VOCs on the microbiological and chemical composition of PBAPs.

Figure S7. Pearson correlation results for the comparison between the gas phase VOCs and the microbiological composition of PBAPs.

References. 


\section{S1 Materials and reagents}

L-Alanine (Ala), L-Aspartic acid (Asp), L-Glutamic acid (Glu), L-Glutamine (Gln), Glycine (Gly), LIsoleucine (Iso), L-Leucine (Leu), L-Lysine (Lys), L-Methionine (Met), L-Phenylalanine (Phe), LProline (Pro), L-Threonine (Thr), L-Tryptophan (Trp), L-Tyrosine (Tyr) and L-Valine (Val) from Seikagaku Kogyo Co. (Tokyo, Japan); L-Arginine (ca. 99 \%) (Arg), L-Histidine ( $\geq 99 \%$ ) (His) and Inositol (ca. 99 \%) from MERCK (Darmstadt, Germany); L-Serine (ca. 99 \%) (Ser) from Ega-Chemie (Steinheim, Germany), Asparagine anhydrous (Asn), Sucrose ( $\geq 99.5 \%$ ), 1,6-Anhydro-b-D-glucose (99 \%), D-Arabitol, D-Mannitol, Glycine-2,2-d 2 (Gly-d2), L-Lysine-4,4,5,5- $\mathrm{d}_{4}$ (98 atom\% D, 98\% (CP)) (Lys-d4) and L-Phenylalanie-3,3-d 2 (98 atom\% D) (Phe-d2) from Sigma-Aldrich (St. Louis, USA); D(+)-Trehalose dihydrate (purity $99 \%$ ) from AK Scientic (Union City, USA) and D-Fructose$13 \mathrm{C}_{6}$ from Carbosynth Ltd. (Berkshire, UK) were used for the preparation of amino acids and saccharides stock solutions. All labelled compounds were used as internal standards (ISTD) in the analysis. N-Hexane (purity 98 \%) from VWR Chemicals (Fontenay-sous-Bois, France) was used for lipid removal from the extracted samples. Formic acid ( $\geq 99 \%)$ from VWR, acetonitrile $(\geq 99.9 \%)$ from Sigma-Aldrich, and ultra-pure water from a Millipore DirectQ-UV system (Molsheim, France) were used for the preparation of the mobile phase used in the HPLC analysis.

Individual standard solutions of the different analytes under study, including ISTD compounds, were prepared in $0.1 \%$ formic acid. Concentrations of stock solutions of the target analytes were $1000 \mu \mathrm{g}$ $\mathrm{mL}^{-1}$, with the exception of Tyr $\left(200 \mu \mathrm{g} \mathrm{mL}^{-1}\right)$, Asp $\left(400 \mu \mathrm{g} \mathrm{mL}^{-1}\right)$ and D-Fructose-13C 6 (5000 $\mathrm{ng} \mathrm{mL}^{-}$ ${ }^{1}$ ). Calibration solutions were prepared daily by dilution of the stock standard solutions with $0.1 \%$ formic acid. Stock standard solutions were stable at $4{ }^{\circ} \mathrm{C}$ for two weeks.

All the tools and glassware used for sample preparation were ultrasonic cleaned, before use, with water and methanol ( $\geq 99 \%$ ) from Honeywell Riedel-de Haën (Charlotte, NC, USA) and dried at $150{ }^{\circ} \mathrm{C}$ in an oven (Heraeus, Hanau, Germany). 


\section{S2. Determination of gas phase volatile organic compounds}

Volatile organic compounds (VOCs) were measured by PTR-MS. The application of a nondissociative proton transfer reaction enables the measurement of most of the common VOCs with a high time-resolution and sensitivity without any sample treatment (Yuan et al., 2017; de Gouw et al., 2003). A disadvantage of PTR-MS is that it only gives information about the molecular mass of protonated compounds but not about their molecular structure. In this study, thirteen masses measured by PTR-MS were identified: 33, 42, 47, 59, 61, 69, 71, 73, 79, 81, 87, 93 and 137 Da. These masses were related to methanol, acetonitrile, ethanol, acetone, acetic acid, isoprene, methacrolein, 2butanone, benzene, $\alpha$-pinene fragment, methylbutenol, toluene and monoterpenes respectively (de Gouw and Warneke, 2007; Taipale et al., 2008). However, some of them might be originating from other VOCs. Sampling was conducted at $8.4 \mathrm{~m}$ above the ground level (inside the canopy) as described by Rantala et al. (2015). PTR-MS was calibrated with a calibration gas standard (Apel-Riemer Environmental, Inc.) containing 1 ppmv of all the VOCs analyzed in this study with the exception of methanol and acetonitrile, which were quantified based on the relative transmission curve. Calibration, the determination of the relative transmission curve and the calculations of volume mixing ratios, were conducted according to procedures described by Taipale et al. (2008). 
Table S1. Aerosol sampling dates and sampling volume.

\begin{tabular}{|c|c|c|c|}
\hline Sample & Start (day and hour) & Stop (day and hour) & Sampling volume $\left[\mathrm{m}^{3}\right]$ \\
\hline Sample 1 (S1) & 04.09.2017 09:07 & 06.09.2017 09:20 & 92.27 \\
\hline Sample 2 (S2) & 06.09.2017 09:20 & 08.09.2017 08:45 & 90.63 \\
\hline Sample 3 (S3) & 11.09.2017 08:24 & 13.09.2017 07:42 & 90.46 \\
\hline Sample 4 (S4) & 13.09.2017 07:42 & 15.09.2017 08:54 & 93.94 \\
\hline Sample 5 (S5) & 18.09.2017 08:35 & 20.09.2017 08:27 & 91.46 \\
\hline Sample 6 (S6) & 20.09.2017 08:27 & 22.09.2017 09:18 & 93.40 \\
\hline Sample 7 (S7) & 25.09.2017 08:35 & 27.09.2017 08:48 & 92.33 \\
\hline Sample 8 (S8) & 27.09.2017 08:48 & 29.09.2017 09:31 & 93.09 \\
\hline Sample 9 (S9) & 02.10.2017 08:10 & 04.10.2017 09:33 & 94.29 \\
\hline Sample 10 (S10) & 04.10.2017 09:33 & $06.10 .201708: 26$ & 89.56 \\
\hline Sample 11 (S11) & 09.10.2017 08:09 & 11.10.2017 08:59 & 93.14 \\
\hline Sample 12 (S12) & 11.10.2017 08:59 & 13.10.2017 09:19 & 92.18 \\
\hline Sample 13 (S13) & 23.10.2017 08:49 & 25.10 .2017 09:03 & 91.99 \\
\hline Sample 14 (S14) & 25.10.2017 09:03 & 27.10.2017 08:49 & 91.27 \\
\hline Sample 15 (S15) & 30.10.2017 09:32 & 01.11.2017 09:19 & 91.12 \\
\hline Sample 16 (S16) & 01.11.2017 09:19 & 03.11.2017 10:09 & 93.11 \\
\hline Sample 17 (S17) & 06.11.2017 10:20 & 08.11.1017 09:35 & 90.20 \\
\hline Sample 18 (S18) & 08.11.2017 09:35 & 10.11.2017 09:58 & 92.35 \\
\hline Sample 19 (S19) & 13.11.2017 10:08 & 15.11 .201710 .56 & 93.11 \\
\hline Sample 20 (S20) & 15.11.2017 10:56 & 17.11.2017 10:23 & 90.49 \\
\hline Sample 21 (S21) & 20.11 .2017 10:05 & 22.11.2017 09:40 & 90.81 \\
\hline
\end{tabular}


Table S2. Ionization conditions for amino acids and saccharides.

\begin{tabular}{cc}
\hline Parameter & Value \\
\hline Gas temperature $\left({ }^{\circ} \mathrm{C}\right)$ & 290 \\
Gas flow $\left(\mathrm{mL} \mathrm{min}^{-1}\right)$ & 9 \\
Nebulizer pressure $(\mathrm{psi})$ & 50 \\
Capillary voltage $(\mathrm{V})$ & 3900 \\
\hline
\end{tabular}


Table S3. Multiple reaction monitoring settings (b) for amino acids and saccharides. MS, precursor ion, MS/MS, product ion F, Fragmentor voltage; $\mathrm{CE}$, collision energy; and CAV, cell accelerator voltage. $*$ Ionized as $[\mathrm{M}+\mathrm{Na}]+$ adduct.

\begin{tabular}{|c|c|c|c|c|c|c|}
\hline Compound & MS ion $(\mathrm{m} / \mathrm{z})$ & $\mathrm{MS}^{2}$ ion $(\mathrm{m} / \mathrm{z})$ & $\overline{F(V)}$ & CE(V) & $\overline{C A V}(\mathrm{~V})$ & Ionization \\
\hline Ala & 90 & 44 & 61 & 9 & 4 & + \\
\hline Arg & 175 & 70 & 61 & 25 & 4 & + \\
\hline Asn & 133 & 74 & 61 & 17 & 4 & + \\
\hline Asp & 134 & 74 & 61 & 13 & 4 & + \\
\hline Glu & 148 & 84 & 61 & 17 & 4 & + \\
\hline Gln & 147 & 84 & 61 & 17 & 4 & + \\
\hline Gly & 76 & 30 & 61 & 9 & 4 & + \\
\hline Gly-d2 & 78 & 32 & 61 & 9 & 4 & + \\
\hline His & 156 & 110 & 61 & 13 & 4 & + \\
\hline Iso & 132 & 69 & 61 & 9 & 4 & + \\
\hline Leu & 132 & 44 & 61 & 25 & 4 & + \\
\hline Lys & 147 & 84 & 61 & 17 & 4 & + \\
\hline Lys-d4 & 151 & 88 & 61 & 17 & 4 & + \\
\hline Met & 150 & 56 & 61 & 17 & 4 & + \\
\hline Phe & 166 & 120 & 61 & 13 & 4 & + \\
\hline Phe-d2 & 168 & 122 & 61 & 13 & 4 & + \\
\hline Pro & 116 & 70 & 61 & 17 & 4 & + \\
\hline Ser & 106 & 60 & 61 & 9 & 4 & + \\
\hline Thr & 120 & 74 & 61 & 9 & 4 & + \\
\hline Trp & 205 & 188 & 61 & 5 & 4 & + \\
\hline Tyr & 182 & 136 & 61 & 13 & 4 & + \\
\hline Val & 118 & 72 & 61 & 9 & 4 & + \\
\hline Sucrose & 365 & 203 & 181 & 40 & 4 & - \\
\hline Trehalose & 365 & 203 & 181 & 40 & 4 & - \\
\hline Arabitol & 151 & 89 & 105 & 15 & 0 & - \\
\hline Fructose & 179 & 89 & 75 & 3 & 0 & - \\
\hline Fructose- $13 \mathrm{C}_{6}$ & 185 & 92 & 75 & 5 & 0 & - \\
\hline Inositol & 179 & 89 & 105 & 20 & 2 & - \\
\hline Levoglucosan* & 185 & - & 61 & 0 & 4 & + \\
\hline Mannitol & 181 & 89 & 105 & 15 & 2 & - \\
\hline Mannose & 179 & 89 & 105 & 5 & 2 & - \\
\hline
\end{tabular}


Table S4. Ancillary data for statistical analysis.

\section{Concentration of atmospheric gases, aerosol, meteorological and environmental parameters}

\begin{tabular}{|c|c|c|c|}
\hline M01 & Total particle concentration $\left(1 \mathrm{~cm}^{-3}\right)$ & M22 & Daily sum precipitation $(\mathrm{mm})$ \\
\hline M02 & Methane flux (nmol m$\left.{ }^{-2} \mathrm{~s}^{-1}\right)$ & M23 & Ultraviolet $\mathrm{A}$ radiation $\left(\mathrm{W} \mathrm{m}^{-2}\right)$ \\
\hline M03 & Evapotranspiration ( $\mathrm{mmol} \mathrm{m}^{-2}$ per half hour) & M24 & Utraviolet $\mathrm{B}$ radiation $\left(\mathrm{W} \mathrm{m}^{-2}\right)$ \\
\hline M04 & Carbon dioxide flux $\left(\mu \mathrm{mol} \mathrm{m} \mathrm{m}^{-2} \mathrm{~s}^{-1}\right)$ & M25 & Global shortwave solar radiation $\left(\mathrm{W} \mathrm{m}^{-2}\right)$ \\
\hline M05 & Gross primary production derived from net ecosystem exchange $\left(\mu \mathrm{mol} \mathrm{m} \mathrm{m}^{-2} \mathrm{~s}^{-1}\right)$ & M26 & Reflected shortwave radiation $\left(\mathrm{W} \mathrm{m}^{-2}\right)$ \\
\hline M06 & $\mathrm{CO}_{2}(\mathrm{ppm})$ & M27 & Reflected photosynth. active radiation $\left(\mu \mathrm{mol} \mathrm{m} \mathrm{m}^{-2} \mathrm{~s}^{-1}\right)$ \\
\hline M07 & Water vapor concentration (ppth) & M28 & Photosynth. active radiation $\left(\mu \mathrm{mol} \mathrm{m} \mathrm{m}^{-2} \mathrm{~s}^{-1}\right)$ \\
\hline M08 & $\mathrm{CO}(\mathrm{ppb})$ & M29 & Diffuse photosynth. active radiation $\left(\mu \mathrm{mol} \mathrm{m} \mathrm{m}^{-2} \mathrm{~s}^{-1}\right)$ \\
\hline M09 & $\mathrm{O}_{3}(\mathrm{ppb})$ & M30 & Below canopy photosynth. active rad. $\left(\mu \mathrm{mol} \mathrm{m} \mathrm{m}^{-2} \mathrm{~s}^{-1}\right)$ \\
\hline M10 & NO (ppb) & M31 & Incoming far-infrared radiation $\left(\mathrm{W} / \mathrm{m}^{-2}\right)$ \\
\hline M11 & $\mathrm{NO}_{\mathrm{x}}(\mathrm{ppb})$ & M32 & Outgoing far-infrared radiation $\left(\mathrm{W} \mathrm{m}^{-2}\right)$ \\
\hline M12 & $\mathrm{SO}_{2}(\mathrm{ppb})$ & M33 & Soil temperature in the organic layer $\left({ }^{\circ} \mathrm{C}\right)$ \\
\hline M13 & Atmospheric pressure $(\mathrm{hPa})$ & M34 & Soil temperature in A horizon $\left({ }^{\circ} \mathrm{C}\right)$ \\
\hline M14 & Air temperature (Celsius) & M35 & Soil temperature in $\mathrm{B} 1$ horizon $\left({ }^{\circ} \mathrm{C}\right)$ \\
\hline M15 & Relative humidity (\%) & M36 & Soil temperature in $\mathrm{B} 2$ horizon $\left({ }^{\circ} \mathrm{C}\right)$ \\
\hline M16 & Horizontal wind speed $\left(\mathrm{m} \mathrm{s}^{-1}\right)$ & M37 & Vol. soil water content in the organic layer $\left(\mathrm{m}^{3} \mathrm{~m}^{-3}\right)$ \\
\hline M17 & Horizontal wind direction (degree) & M38 & Vol. soil water content in A horizon $\left(\mathrm{m}^{3} \mathrm{~m}^{-3}\right)$ \\
\hline M18 & Wind direction, average of above-canopy (degree) & M39 & Volumetric soil water content in B1 horizon $\left(\mathrm{m}^{3} \mathrm{~m}^{-3}\right)$ \\
\hline M19 & Visibility (km) & M40 & Volumetric soil water content in B2 horizon $\left(\mathrm{m}^{3} \mathrm{~m}^{-3}\right)$ \\
\hline M20 & Cloud base height layer $1(\mathrm{~m})$ & M41 & Soil heat flux ( $\left.\mathrm{W} \mathrm{m}^{-2}\right)$ \\
\hline M21 & Daily sum of snowfall accumulated (mm) & & \\
\hline
\end{tabular}


Table S5. Analytical features of the method used for the chemical characterization of PBAPs.

\begin{tabular}{|c|c|c|c|c|c|}
\hline Compound & Calibration & $\mathbf{R}^{2}$ & $\begin{array}{c}\text { LOD } \\
\left(\mathrm{ng} \mathrm{m}^{-3}\right)\end{array}$ & $\begin{array}{c}\text { LOQ } \\
\left(\mathrm{ng} \mathrm{m}^{-3}\right)\end{array}$ & $\begin{array}{c}\text { Linearity } \\
\left(\mathrm{ng} \mathrm{m}^{-3}\right)\end{array}$ \\
\hline Ala & $y=0.0014 x+0.0265$ & 0.992 & 0.01 & 0.03 & $0.03-20.82$ \\
\hline Arg & $\mathrm{y}=0.0114 \mathrm{x}+0.0938$ & 0.996 & 0.01 & 0.02 & $0.02-25.09$ \\
\hline Asn & $y=0.0018 x-0.0054$ & 0.999 & 0.02 & 0.05 & $0.05-11.41$ \\
\hline Asp & $y=0.0037 x-0.0032$ & 0.997 & 0.01 & 0.03 & $0.03-18.55$ \\
\hline Glu & $y=0.007 x-0.0026$ & 0.999 & 0.01 & 0.03 & $0.03-23.83$ \\
\hline Gln & $y=0.0067 x-0.0007$ & 0.999 & 0.01 & 0.03 & $0.03-35.02$ \\
\hline Gly & $y=0.0024 x+0.0041$ & 0.999 & 0.01 & 0.03 & $0.03-9.33$ \\
\hline His & $y=0.0167 x+0.0903$ & 0.998 & 0.04 & 0.12 & $0.12-11.40$ \\
\hline Iso & $y=0.0002 x+0.0016$ & 0.997 & 0.01 & 0.03 & $0.03-2.64$ \\
\hline Leu & $y=0.0001 x-0.0008$ & 0.996 & 0.01 & 0.03 & $0.03-12.91$ \\
\hline Lys & $y=0.007 x+0.0691$ & 0.999 & 0.01 & 0.03 & $0.03-3.40$ \\
\hline Met & $y=0.0006 x+0.0077$ & 0.996 & 0.01 & 0.03 & $0.02-11.41$ \\
\hline Phe & $y=0.0018 x+0.0002$ & 0.999 & 0.01 & 0.03 & $0.03-3.50$ \\
\hline Pro & $y=0.0035 x+0.0667$ & 0.993 & 0.01 & 0.03 & $0.03-8.41$ \\
\hline Ser & $y=0.0078 x-0.0126$ & 0.999 & 0.03 & 0.10 & $0.10-9.33$ \\
\hline Thr & $y=0.0038 x+0.0011$ & 0.999 & 0.01 & 0.02 & $0.02-12.39$ \\
\hline $\operatorname{Trp}$ & $y=0.0008 x+0.0036$ & 0.999 & 0.01 & 0.03 & $0.03-0.34$ \\
\hline Tyr & $y=0.0004 x+0.0045$ & 0.997 & 0.01 & 0.03 & $0.03-5.73$ \\
\hline Val & $y=0.0033 x+0.0705$ & 0.991 & 0.01 & 0.03 & $0.03-3.85$ \\
\hline Arabitol & $y=0.00005 x+0.0005$ & 0.999 & 0.05 & 0.16 & $0.16-64.15$ \\
\hline Fructose & $y=0.0026 x-0.0117$ & 0.998 & 0.01 & 0.01 & $0.02-122.72$ \\
\hline Inositol & $y=0.002 x+0.0187$ & 0.996 & 0.01 & 0.03 & $0.03-1.35$ \\
\hline Levoglucosan & $y=0.0013 x+0.0261$ & 0.995 & 0.03 & 0.10 & $0.10-64.15$ \\
\hline Mannitol & $y=0.004 x-0.0011$ & 0.998 & 0.02 & 0.06 & $0.06-47.36$ \\
\hline Mannose & $y=0.0024 x-0.0048$ & 0.999 & 0.01 & 0.03 & $0.03-8.83$ \\
\hline Sucrose & $y=0.0023 x+0.0147$ & 0.998 & 0.02 & 0.06 & $0.06-25.77$ \\
\hline Trehalose & $y=0.004 x-0.0011$ & 0.995 & 0.01 & 0.04 & $0.04-14.36$ \\
\hline
\end{tabular}


Table S6. Extraction recoveries of the method used for the chemical characterization of PBAPs.

\begin{tabular}{lccc}
\hline Compounds & Added $\left.\mathbf{n g} \mathbf{~ m L}^{-\mathbf{1}}\right)$ & Found $\left.\mathbf{( n g} \mathbf{~ m L}^{-\mathbf{1}}\right)$ & Recovery (\%) \\
\hline Ala & 250 & 261.5 & 104.6 \\
Arg & 250 & 260.6 & 104.2 \\
Asn & 250 & 295.2 & 118.1 \\
Asp & 250 & 289.9 & 115.9 \\
Glu & 250 & 238.9 & 95.6 \\
Gln & 250 & 278.0 & 111.2 \\
Gly & 250 & 238.9 & 95.6 \\
His & 250 & 254.9 & 101.9 \\
Iso & 250 & 215.3 & 86.1 \\
Leu & 250 & 226.0 & 90.4 \\
Lys & 250 & 246.0 & 98.4 \\
Met & 250 & 206.8 & 82.7 \\
Phe & 250 & 215.2 & 86.1 \\
Pro & 250 & 231.7 & 92.7 \\
Ser & 250 & 334.9 & 133.9 \\
Thr & 250 & 194.9 & 77.9 \\
Trp & 250 & 183.1 & 73.2 \\
Tyr & 250 & 215.3 & 86.1 \\
Val & 250 & 219.9 & 87.9 \\
Arabitol & 250 & 219.8 & 87.9 \\
Fructose & 250 & 258.9 & 104.5 \\
Inositol & 2500 & 2387.8 & 95.5 \\
Levoglucosan & 250 & 313.5 & 125.4 \\
Mannitol & 125 & 105.1 & 84.1 \\
Mannose & 250 & 211.9 & 84.8 \\
Sucrose & 125 & 124.4 & 99.5 \\
Trehalose & 250 & 248.1 & 99.3 \\
\hline
\end{tabular}


Table S7. Effect of the sample matrix on the ionization of the different amino acids and saccharides analyzed by the method used for the chemical characterization of PBAPs.

\begin{tabular}{lcccc}
\hline Compounds & $\begin{array}{c}\text { Recovery } \\
\text { L1 }(\mathbf{\%})\end{array}$ & $\begin{array}{c}\text { Recovery } \\
\text { L2 }(\mathbf{\%})\end{array}$ & $\begin{array}{c}\text { Recovery } \\
\text { L3 }(\boldsymbol{\%})\end{array}$ & $\begin{array}{c}\text { Average } \\
\text { Recovery }(\%)\end{array}$ \\
\hline Ala & 110.5 & 102.2 & 123.0 & 111.9 \\
Arg & 88.5 & 119.5 & 126.9 & 111.6 \\
Asn & 134.2 & 94.8 & 120.1 & 116.4 \\
Asp & 84.5 & 100.2 & 122.3 & 102.4 \\
Gln & 114.3 & 88.3 & 78.0 & 93.5 \\
Glu & 85.6 & 78.3 & 119.2 & 94.4 \\
Gly & 89.3 & 104.0 & 126.7 & 106.7 \\
His & 94.0 & 112.4 & 115.9 & 107.4 \\
Iso & 111.7 & 116.5 & 117.6 & 115.3 \\
Leu & 70.5 & 110.3 & 117.4 & 99.4 \\
Lys & 117.3 & 55.3 & 126.3 & 99.7 \\
Met & 78.2 & 125.9 & 116.2 & 106.8 \\
Phe & 83.1 & 118.3 & 117.8 & 106.4 \\
Pro & 79.2 & 100.0 & 116.7 & 98.7 \\
Ser & 105.4 & 92.4 & 132.7 & 110.1 \\
Thr & 72.8 & 97.7 & 122.1 & 97.5 \\
Trp & 87.6 & 114.4 & 116.9 & 106.3 \\
Tyr & 79.8 & 97.1 & 118.8 & 98.6 \\
Val & 80.7 & 139.2 & 117.5 & 112.5 \\
Arabitol & 78.3 & 79.1 & 93.7 & 83.7 \\
Fructose & 90.1 & 73.3 & 77.5 & 80.3 \\
Inositol & 77.1 & 93.1 & 128.7 & 99.6 \\
Mannitol & 74.2 & 86.6 & 83.5 & 81.5 \\
Mannose & 81.2 & 70.3 & 108.3 & 86.6 \\
Sucrose & 78.7 & 87.3 & 128.6 & 98.2 \\
Trehalose & 79.8 & 86.1 & 118.8 & 94.9 \\
\hline
\end{tabular}

L1, addition of $8 \mathrm{ng}$ from each compound; L2, addition of $77 \mathrm{ng}$ from each compound; L3, addition of $111 \mathrm{ng}$ from each compound. 
Table S8. Summary statistics for the determination of amino acids in PBAPs. N, Number of observations; Av., Average concentration $\left(\mathrm{ng} \mathrm{m}^{-3}\right)$; SD, Standard deviation ( $\left.\mathrm{ng} \mathrm{m}^{-3}\right)$; CV, Coefficient of variation (\%); Min., Minimum (ng $\left.\mathrm{m}^{-3}\right)$; Max, Maximum $\left(\mathrm{ng} \mathrm{m}^{-3}\right)$.

\begin{tabular}{|c|c|c|c|c|c|c|c|c|c|c|}
\hline & Size & $\mathbf{N}$ & Av. & SD & $\mathrm{CV}$ & Min & Max & Range & $\begin{array}{c}\text { Std. } \\
\text { Skewness* }\end{array}$ & $\begin{array}{c}\text { Std. } \\
\text { Kurtosis* }\end{array}$ \\
\hline \multirow{4}{*}{ Ala } & PM 1 & 21 & 0.5 & 1.3 & 37.4 & 0.3 & 0.9 & 0.6 & 0.1 & -0.3 \\
\hline & PM 2.5 & 15 & 0.5 & 1.3 & 34.4 & 0.3 & 0.8 & 0.5 & -0.3 & -0.3 \\
\hline & PM10 & 18 & 1.8 & 1.4 & 57.8 & 1.1 & 3.0 & 2.0 & -0.6 & -1.1 \\
\hline & $\mathrm{PM}>10$ & 18 & 1.2 & 1.9 & 153.1 & 0.4 & 3.7 & 3.4 & -0.2 & -0.4 \\
\hline \multirow[t]{4}{*}{ Arg } & PM 1 & 11 & 0.4 & 1.1 & 8.5 & 0.4 & 0.5 & 0.1 & -1.0 & -0.1 \\
\hline & PM 2.5 & 16 & 0.7 & 1.5 & 98.3 & 0.3 & 1.2 & 0.9 & -1.9 & 0.3 \\
\hline & PM10 & 20 & 1.9 & 1.5 & 67.5 & 0.6 & 3.6 & 3.0 & -1.7 & 1.4 \\
\hline & $\mathrm{PM}>10$ & 20 & 1.3 & 1.9 & 120.3 & 0.4 & 4.1 & 3.6 & -0.9 & -0.5 \\
\hline \multirow[t]{4}{*}{ Asn } & PM 1 & & & & & & & & & \\
\hline & PM 2.5 & & & & & & & & & \\
\hline & PM10 & 17 & 1.1 & 1.7 & 165.3 & 0.5 & 2.0 & 1.5 & -1.2 & -0.9 \\
\hline & $\mathrm{PM}>10$ & 21 & 0.7 & 1.9 & 159.2 & 0.2 & 2.9 & 2.7 & 0.5 & -0.2 \\
\hline \multirow[t]{4}{*}{ Asp } & PM 1 & & & & & & & & & \\
\hline & PM 2.5 & & & & & & & & & \\
\hline & PM10 & 10 & 1.6 & 1.4 & 61.2 & 1.0 & 2.5 & 1.5 & -0.5 & -0.5 \\
\hline & $\mathrm{PM}>10$ & 7 & 1.6 & 1.5 & 90.2 & 1.0 & 3.6 & 2.5 & 1.7 & 1.7 \\
\hline \multirow[t]{4}{*}{ Gln } & PM 1 & 12 & 0.5 & 1.3 & 38.1 & 0.3 & 0.8 & 0.5 & 0.2 & -0.4 \\
\hline & PM 2.5 & 19 & 0.8 & 1.7 & 94.9 & 0.3 & 1.3 & 1.0 & -1.2 & -1.0 \\
\hline & PM10 & 20 & 1.9 & 1.9 & 100.5 & 0.4 & 3.9 & 3.5 & -1.9 & 0.4 \\
\hline & $\mathrm{PM}>10$ & 21 & 1.3 & 1.8 & 132.1 & 0.4 & 4.7 & 4.3 & -0.3 & 0.3 \\
\hline \multirow[t]{4}{*}{ Glu } & PM 1 & 20 & 0.4 & 1.3 & 82.4 & 0.3 & 0.6 & 0.4 & 0.5 & -0.4 \\
\hline & PM 2.5 & 18 & 0.6 & 1.5 & 24.5 & 0.3 & 1.0 & 0.7 & -1.3 & -0.8 \\
\hline & PM10 & 20 & 1.7 & 1.5 & 76.9 & 0.7 & 2.7 & 2.0 & -1.3 & -0.8 \\
\hline & $\mathrm{PM}>10$ & 21 & 1.1 & 1.8 & 171.6 & 0.5 & 4.0 & 3.5 & 0.7 & -0.3 \\
\hline \multirow[t]{4}{*}{ Gly } & PM 1 & 17 & 0.7 & 1.5 & 144.7 & 0.3 & 1.6 & 1.3 & -1.4 & 1.1 \\
\hline & PM 2.5 & & & & & & & & & \\
\hline & PM10 & 8 & 1.0 & 1.5 & 188.9 & 0.6 & 1.6 & 1.1 & 0.0 & -1.2 \\
\hline & $\mathrm{PM}>10$ & 13 & 0.9 & 1.8 & 191.3 & 0.4 & 2.6 & 2.2 & 0.6 & -0.8 \\
\hline \multirow[t]{4}{*}{ His } & PM 1 & & & & & & & & & \\
\hline & PM 2.5 & 11 & 0.3 & 1.1 & 8.4 & 0.3 & 0.4 & 0.1 & -0.5 & -1.2 \\
\hline & PM10 & 11 & 1.5 & 1.3 & 65.4 & 0.9 & 2.0 & 1.1 & -1.2 & -0.3 \\
\hline & $\mathrm{PM}>10$ & 13 & 1.2 & 1.6 & 137.4 & 0.5 & 2.7 & 2.2 & -0.6 & -0.3 \\
\hline \multirow[t]{4}{*}{ Ile } & PM 1 & & & & & & & & & \\
\hline & PM 2.5 & & & & & & & & & \\
\hline & PM10 & 17 & 0.7 & 1.3 & 81.4 & 0.5 & 1.1 & 0.6 & 0.4 & -1.2 \\
\hline & $\mathrm{PM}>10$ & 11 & 0.7 & 1.4 & 119.5 & 0.4 & 1.5 & 1.1 & 0.4 & 0.7 \\
\hline \multirow[t]{3}{*}{ Leu } & PM 1 & & & & & & & & & \\
\hline & PM 2.5 & & & & & & & & & \\
\hline & PM10 & 18 & 2.0 & 1.5 & 58.4 & 0.9 & 3.0 & 2.2 & -1.1 & -0.7 \\
\hline
\end{tabular}




\begin{tabular}{|c|c|c|c|c|c|c|c|c|c|c|}
\hline \multirow{3}{*}{ Lys } & $\mathrm{PM}>10$ & 17 & 1.7 & 1.4 & 67.1 & 0.9 & 2.9 & 2.0 & -0.2 & -0.8 \\
\hline & PM 1 & & & & & & & & & \\
\hline & PM 2.5 & & & & & & & & & \\
\hline \multirow{6}{*}{ Phe } & PM10 & 13 & 0.9 & 1.2 & 101.9 & 0.7 & 1.2 & 0.5 & 1.6 & 0.4 \\
\hline & $\mathrm{PM}>10$ & 13 & 0.7 & 1.6 & 127.1 & 0.3 & 1.7 & 1.4 & 0.0 & -0.1 \\
\hline & PM 1 & & & & & & & & & \\
\hline & PM 2.5 & 14 & 0.3 & 1.5 & 27.9 & 0.1 & 0.6 & 0.5 & 0.9 & 0.7 \\
\hline & PM10 & 19 & 0.6 & 1.8 & 57.4 & 0.2 & 1.2 & 1.0 & -1.5 & -0.4 \\
\hline & $\mathrm{PM}>10$ & 18 & 0.4 & 2.0 & 81.6 & 0.1 & 1.7 & 1.6 & -0.1 & -0.6 \\
\hline \multirow[t]{4}{*}{ Pro } & PM 1 & 15 & 0.3 & 1.3 & 21.4 & 0.2 & 0.5 & 0.3 & 0.1 & -0.8 \\
\hline & PM 2.5 & 14 & 0.3 & 1.3 & 22.9 & 0.2 & 0.4 & 0.3 & -0.8 & -0.8 \\
\hline & PM10 & 18 & 1.2 & 1.3 & 96.9 & 0.5 & 1.7 & 1.2 & -1.6 & 1.1 \\
\hline & $\mathrm{PM}>10$ & 21 & 1.2 & 1.7 & 177.9 & 0.4 & 2.5 & 2.1 & -0.4 & -0.9 \\
\hline \multirow[t]{4}{*}{ Ser } & PM 1 & & & & & & & & & \\
\hline & PM 2.5 & & & & & & & & & \\
\hline & PM10 & 20 & 1.7 & 1.3 & 57.8 & 0.9 & 2.6 & 1.7 & -1.0 & -0.5 \\
\hline & $\mathrm{PM}>10$ & 16 & 1.3 & 1.6 & 74.6 & 0.5 & 2.3 & 1.8 & -0.7 & -0.4 \\
\hline \multirow[t]{4}{*}{ Thr } & PM 1 & & & & & & & & & \\
\hline & PM 2.5 & & & & & & & & & \\
\hline & PM10 & 20 & 1.4 & 1.4 & 116.3 & 0.7 & 2.2 & 1.5 & -0.8 & -1.1 \\
\hline & $\mathrm{PM}>10$ & 19 & 0.8 & 2.0 & 131.7 & 0.2 & 3.0 & 2.8 & -0.6 & -0.1 \\
\hline \multirow[t]{4}{*}{ Trp } & PM 1 & & & & & & & & & \\
\hline & PM 2.5 & & & & & & & & & \\
\hline & PM10 & 11 & 0.4 & 1.2 & 25.1 & 0.3 & 0.6 & 0.3 & -1.4 & 0.5 \\
\hline & $\mathrm{PM}>10$ & 10 & 0.4 & 1.5 & 36.2 & 0.2 & 0.6 & 0.4 & -0.5 & -0.1 \\
\hline \multirow[t]{4}{*}{ Tyr } & PM 1 & & & & & & & & & \\
\hline & PM 2.5 & & & & & & & & & \\
\hline & PM10 & 14 & 0.9 & 1.2 & 52.5 & 0.7 & 1.2 & 0.6 & -0.4 & -0.9 \\
\hline & $\mathrm{PM}>10$ & 15 & 0.8 & 1.6 & 78.8 & 0.3 & 2.1 & 1.9 & -0.1 & 1.5 \\
\hline \multirow[t]{3}{*}{ Val } & PM 1 & 18 & 0.3 & 1.2 & 14.7 & 0.3 & 0.5 & 0.2 & 1.2 & 0.4 \\
\hline & PM 2.5 & & & & & & & & & \\
\hline & PM10 & 13 & 0.5 & 1.5 & 64.1 & 0.2 & 0.8 & 0.6 & -1.7 & 0.1 \\
\hline
\end{tabular}

* Calculated after logarithmic transformation of the original data 
Table S9. Summary statistics for the determination of saccharides in PBAPs. N, Number of observations; Av., Average concentration $\left(\mathrm{ng} \mathrm{m}^{-3}\right)$; SD, Standard deviation ( $\left.\mathrm{ng} \mathrm{m}^{-3}\right)$; CV, Coefficient of variation (\%); Min., Minimum ( $\left.\mathrm{ng} \mathrm{m}^{-3}\right)$; Max, Maximum $\left(\mathrm{ng} \mathrm{m}^{-3}\right)$.

\begin{tabular}{|c|c|c|c|c|c|c|c|c|c|c|}
\hline & Size & $\mathbf{N}$ & Av. & SD & $\mathrm{CV}$ & Min & $\operatorname{Max}$ & Range & $\begin{array}{c}\text { Std. } \\
\text { skewness* }\end{array}$ & $\begin{array}{c}\text { Std. } \\
\text { kurtosis* }\end{array}$ \\
\hline \multirow{4}{*}{$\begin{array}{l}\text { Arabitol } \\
\text { (Ara) }\end{array}$} & PM 1 & 10 & 0.9 & 1.7 & 58.4 & 0.4 & 2.7 & 2.3 & -0.3 & 0.4 \\
\hline & PM 2.5 & 18 & 1.4 & 1.8 & 41.5 & 0.4 & 3.8 & 3.4 & -0.6 & -1.1 \\
\hline & PM10 & 15 & 4.4 & 1.7 & 33.7 & 1.8 & 10.4 & 8.6 & -1.8 & 0.6 \\
\hline & $\mathrm{PM}>10$ & 9 & 2.3 & 1.3 & 35.2 & 1.2 & 3.7 & 2.4 & -0.9 & 0.0 \\
\hline \multirow{4}{*}{$\begin{array}{l}\text { Fructose } \\
\text { (Fru) }\end{array}$} & PM 1 & & 0.7 & 2.0 & 192.5 & 0.1 & 2.0 & 1.9 & & \\
\hline & PM 2.5 & & 0.9 & 2.1 & 197.3 & 0.1 & 2.8 & 2.7 & & \\
\hline & PM10 & 16 & 6.0 & 1.8 & 32.1 & 1.9 & 13.5 & 11.6 & 0.7 & 1.3 \\
\hline & $\mathrm{PM}>10$ & 13 & 1.3 & 1.6 & 170.7 & 0.6 & 2.9 & 2.3 & 0.7 & 1.3 \\
\hline \multirow{4}{*}{$\begin{array}{l}\text { Inositol } \\
\text { (Ino) }\end{array}$} & PM 1 & & 0.2 & 1.5 & 28.5 & 0.1 & 0.5 & 0.4 & & \\
\hline & PM 2.5 & & 0.3 & 1.6 & 41.5 & 0.1 & 0.5 & 0.4 & & \\
\hline & PM10 & 15 & 1.3 & 1.3 & 115.0 & 1.0 & 2.1 & 1.2 & -0.6 & -0.2 \\
\hline & $\mathrm{PM}>10$ & 14 & 1.4 & 1.4 & 105.5 & 0.9 & 2.2 & 1.3 & 1.6 & 0.8 \\
\hline \multirow{4}{*}{$\begin{array}{l}\text { Mannitol } \\
\text { (Mnt) }\end{array}$} & PM 1 & 10 & 0.9 & 1.7 & 58.4 & 0.4 & 2.7 & 2.3 & 1.3 & 1.2 \\
\hline & PM 2.5 & 18 & 1.4 & 1.8 & 41.5 & 0.4 & 3.8 & 3.4 & -1.0 & -1.1 \\
\hline & PM10 & 20 & 4.4 & 1.7 & 33.7 & 1.8 & 10.4 & 8.6 & -1.1 & -0.2 \\
\hline & $\mathrm{PM}>10$ & 21 & 2.3 & 1.3 & 35.2 & 1.2 & 3.7 & 2.4 & -0.2 & -0.3 \\
\hline \multirow{4}{*}{$\begin{array}{l}\text { Mannose } \\
\text { (Mns) }\end{array}$} & PM 1 & & 0.7 & 2.0 & 192.5 & 0.1 & 2.0 & 1.9 & & \\
\hline & PM 2.5 & & 0.9 & 2.1 & 197.3 & 0.1 & 2.8 & 2.7 & & \\
\hline & PM10 & 16 & 6.0 & 1.8 & 32.1 & 1.9 & 13.5 & 11.6 & -1.6 & -0.3 \\
\hline & $\mathrm{PM}>10$ & 15 & 1.3 & 1.6 & 170.7 & 0.6 & 2.9 & 2.3 & -1.4 & 0.9 \\
\hline \multirow{4}{*}{$\begin{array}{l}\text { Sucrose } \\
\text { (Suc) }\end{array}$} & PM 1 & & 0.2 & 1.5 & 28.5 & 0.1 & 0.5 & 0.4 & & \\
\hline & PM 2.5 & & 0.3 & 1.6 & 41.5 & 0.1 & 0.5 & 0.4 & & \\
\hline & PM10 & 20 & 1.3 & 1.3 & 115.0 & 1.0 & 2.1 & 1.2 & -1.5 & -0.1 \\
\hline & $\mathrm{PM}>10$ & 17 & 1.4 & 1.4 & 105.5 & 0.9 & 2.2 & 1.3 & 0.3 & -0.3 \\
\hline \multirow{4}{*}{$\begin{array}{l}\text { Trehalose } \\
\text { (Tre) }\end{array}$} & PM 1 & & 0.9 & 1.7 & 58.4 & 0.4 & 2.7 & 2.3 & & \\
\hline & PM 2.5 & 17 & 1.4 & 1.8 & 41.5 & 0.4 & 3.8 & 3.4 & -0.8 & -1.0 \\
\hline & PM10 & 11 & 4.4 & 1.7 & 33.7 & 1.8 & 10.4 & 8.6 & 0.2 & -1.0 \\
\hline & $\mathrm{PM}>10$ & 19 & 2.3 & 1.3 & 35.2 & 1.2 & 3.7 & 2.4 & -1.1 & -0.8 \\
\hline
\end{tabular}

* Calculated after logarithmic transformation of the original data. 
Table S10. Summary statistics for the determination of number of gene copies for microbes in PBAPs. N, Number of observations; Av., Average number of gene copies (gene copies $\mathrm{m}^{-3}$ ); SD, Standard deviation (gene copies $\mathrm{m}^{-3}$ ); CV, Coefficient of variation (\%); Min., Minimum (gene copies $\mathrm{m}^{-3}$ ); Max, Maximum (gene copies $\mathrm{m}^{-3}$ ).

\begin{tabular}{lcccccccccc}
\hline & Size & N & Av. & SD & CV & Min & Max & Range & Std. skewness* & Std. kurtosis* \\
\hline Bacteria & PM 1 & 21 & 0.9 & 1.7 & 58.4 & 0.4 & 2.7 & 2.3 & 1.0 & -0.7 \\
(Bac) & PM 2.5 & 21 & 1.4 & 1.8 & 41.5 & 0.4 & 3.8 & 3.4 & 0.2 & -0.7 \\
& PM10 & 21 & 4.4 & 1.7 & 33.7 & 1.8 & 10.4 & 8.6 & -0.5 & -0.8 \\
Fungi & PM $>10$ & 20 & 2.3 & 1.3 & 35.2 & 1.2 & 3.7 & 2.4 & -0.2 & -0.5 \\
(Fun) & PM 1 & 20 & 0.7 & 2.0 & 192.5 & 0.1 & 2.0 & 1.9 & -1.2 & 0.2 \\
& PM 2.5 & 21 & 0.9 & 2.1 & 197.3 & 0.1 & 2.8 & 2.7 & -1.7 & 0.7 \\
Pseudomonas & PM10 & 21 & 6.0 & 1.8 & 32.1 & 1.9 & 13.5 & 11.6 & -0.6 & -0.8 \\
(Pse) & PM $>10$ & 20 & 1.3 & 1.6 & 170.7 & 0.6 & 2.9 & 2.3 & -0.2 & -1.2 \\
& PM 1 & 10 & 0.2 & 1.5 & 28.5 & 0.1 & 0.5 & 0.4 & 0.1 & -0.2 \\
& PM 2.5 & 11 & 0.3 & 1.6 & 41.5 & 0.1 & 0.5 & 0.4 & -0.8 & -0.6 \\
& PM10 & 8 & 1.3 & 1.3 & 115.0 & 1.0 & 2.1 & 1.2 & 0.9 & -0.6 \\
\hline & PM $>10$ & 7 & 1.4 & 1.4 & 105.5 & 0.9 & 2.2 & 1.3 & 0.1 & -1.1 \\
\hline
\end{tabular}

* Calculated after logarithmic transformation of the original data. 
Table S11. Summary statistics for the determination of total DNA in PBAPs. N, Number of observations; Av., Average concentration $\left(\mathrm{ng} \mathrm{m}^{-3}\right)$; SD, Standard deviation ( $\left.\mathrm{ng} \mathrm{m}^{-3}\right)$; CV, Coefficient of variation (\%); Min., Minimum ( $\left.\mathrm{ng} \mathrm{m}^{-3}\right)$; Max, Maximum $\left(\mathrm{ng} \mathrm{m}^{-3}\right)$.

\begin{tabular}{|c|c|c|c|c|c|c|c|c|c|c|}
\hline & Size & $\mathbf{N}$ & Aver. & SD & $\mathrm{CV}$ & Min & Max & Range & Std. skewness* & Std. kurtosis* \\
\hline \multirow[t]{4}{*}{ DNA } & PM 1 & & & & & & & & & \\
\hline & PM 2.5 & 11 & 0.2 & 1.2 & 9.8 & 0.1 & 0.2 & 0.1 & 0.2 & -1.1 \\
\hline & PM10 & 21 & 0.2 & 1.2 & 8.5 & 0.1 & 0.2 & 0.1 & -1.0 & 1.4 \\
\hline & $\mathrm{PM}>10$ & 21 & 0.2 & 1.2 & 11.1 & 0.1 & 0.2 & 0.1 & 1.5 & -0.3 \\
\hline
\end{tabular}

* Calculated after logarithmic transformation of the original data. 
Table S12. Membership table for the cluster classification of the samples based on variations in atmospheric gases concentration, aerosol, meteorological and environmental parameters.

\begin{tabular}{lcccc}
\hline & \multicolumn{4}{c}{ Number of clusters (N) and membership } \\
Sample & $\mathbf{N}=\mathbf{2}$ & $\mathbf{N}=\mathbf{3}$ & $\mathbf{N}=\mathbf{4}$ & $\mathbf{N}=\mathbf{5}$ \\
\hline S1 & 1 & 1 & 1 & 1 \\
S2 & 1 & 1 & 1 & 1 \\
S3 & 1 & 2 & 2 & 2 \\
S4 & 1 & 2 & 2 & 2 \\
S5 & 1 & 2 & 3 & 3 \\
S6 & 1 & 2 & 3 & 3 \\
S7 & 1 & 2 & 2 & 2 \\
S8 & 1 & 2 & 2 & 2 \\
S9 & 1 & 2 & 2 & 2 \\
S10 & 1 & 2 & 2 & 2 \\
S11 & 1 & 2 & 2 & 2 \\
S12 & 1 & 2 & 2 & 2 \\
S13 & 2 & 3 & 3 & 3 \\
S14 & 2 & 3 & 3 & 3 \\
S15 & 2 & 3 & 3 & 3 \\
S16 & 2 & 3 & 3 & 3 \\
S17 & 2 & 3 & 3 & 3 \\
S18 & 2 & 3 & 3 & 3 \\
S19 & 2 & 3 & 3 & 3 \\
S20 & 2 & 3 & 3 & 3 \\
S21 & 2 & 3 & 3 & 5 \\
\hline
\end{tabular}


Table S13. Statistical features of the MLR models used for the potential elucidation of chemical signals from microbes in aerosol particles. $\mathrm{N}$, number of samples in the model; R2, correlation coefficient adjusted by degree of freedom; SEE, standard error of the estimate; MAE, mean absolute error; and D-W, Durbin Watson statistic P-value.

\begin{tabular}{lllccccc}
\hline & Size & N & P-value & R2 & SEE & MAE & D-W \\
\hline Bacteria & PM 1 & 19 & 0.001 & 0.91 & 0.15 & 0.09 & 0.40 \\
& PM 2.5 & 19 & 0.021 & 0.98 & 0.09 & 0.02 & 0.11 \\
& PM10 & 18 & 0.008 & 0.95 & 0.17 & 0.07 & 0.50 \\
Total DNA & PM $>$ 10 & 18 & 0.007 & 0.99 & 0.03 & 0.01 & 0.91 \\
& PM 1 & 19 & 0.001 & 0.92 & 0.27 & 0.12 & 0.13 \\
& PM 2.5 & 19 & 0.002 & 0.96 & 0.19 & 0.07 & 0.39 \\
& PM10 & 19 & 0.002 & 0.96 & 0.02 & 0.01 & 0.33 \\
Fungi & PM $>10$ & 18 & 0.023 & 0.97 & 0.03 & 0.01 & 0.19 \\
& PM 1 & 19 & 0.001 & 0.97 & 0.12 & 0.04 & 1.00 \\
& PM 2.5 & 19 & 0.007 & 0.92 & 0.21 & 0.07 & 0.27 \\
& PM10 & 19 & 0.008 & 0.94 & 0.26 & 0.17 & 0.86 \\
& PM >10 & 19 & 0.005 & 0.97 & 0.08 & 0.02 & 0.60 \\
& PM 1 & 18 & 0.004 & 0.95 & 0.29 & 0.13 & 0.06 \\
& PM 2.5 & 18 & 0.005 & 0.98 & 0.09 & 0.03 & 0.30 \\
& PM10 & 19 & 0.002 & 0.99 & 0.01 & 0.01 & 0.20 \\
& PM $>10$ & 18 & 0.017 & 0.99 & 0.01 & 0.01 & 0.56 \\
\hline
\end{tabular}


Table S14. Statistical features of the MLR models used for the elucidation of potential connections between gas phase VOCs and the microbiological composition of the aerosol particles. N, number of samples in the model; R2, correlation coefficient adjusted by degree of freedom; SEE, standard error of the estimate; MAE, mean absolute error; and D-W, Durbin Watson statistic P-value.

\begin{tabular}{lllccccc}
\hline & Size & N & P-value & $\mathbf{R}^{\mathbf{2}}$ & SEE & MAE & D-W \\
\hline Bacteria & PM 1 & 18 & 0.002 & 0.92 & 0.13 & 0.06 & 0.28 \\
& PM 2.5 & 18 & 0.028 & 0.95 & 0.12 & 0.05 & 0.50 \\
& PM10 & 18 & 0.003 & 0.95 & 0.11 & 0.04 & 0.18 \\
Fungi & PM $>$ 10 & 20 & 0.004 & 0.96 & 0.11 & 0.05 & 0.50 \\
& PM 1 & 19 & 0.001 & 0.94 & 0.16 & 0.06 & 0.43 \\
& PM 2.5 & 18 & 0.006 & 0.93 & 0.20 & 0.07 & 0.41 \\
Pseudomonas & PM10 & 19 & 0.003 & 0.92 & 0.17 & 0.07 & 0.34 \\
& PM $>10$ & 18 & 0.003 & 0.98 & 0.06 & 0.02 & 0.46 \\
& PM 1 & 18 & 0.016 & 0.90 & 0.24 & 0.10 & 0.13 \\
& PM 2.5 & 18 & 0.041 & 0.93 & 0.17 & 0.05 & 0.21 \\
& PM10 & 18 & 0.002 & 0.98 & 0.02 & 0.01 & 0.07 \\
& PM >10 & 18 & 0.004 & 0.94 & 0.05 & 0.02 & 0.37 \\
\hline
\end{tabular}




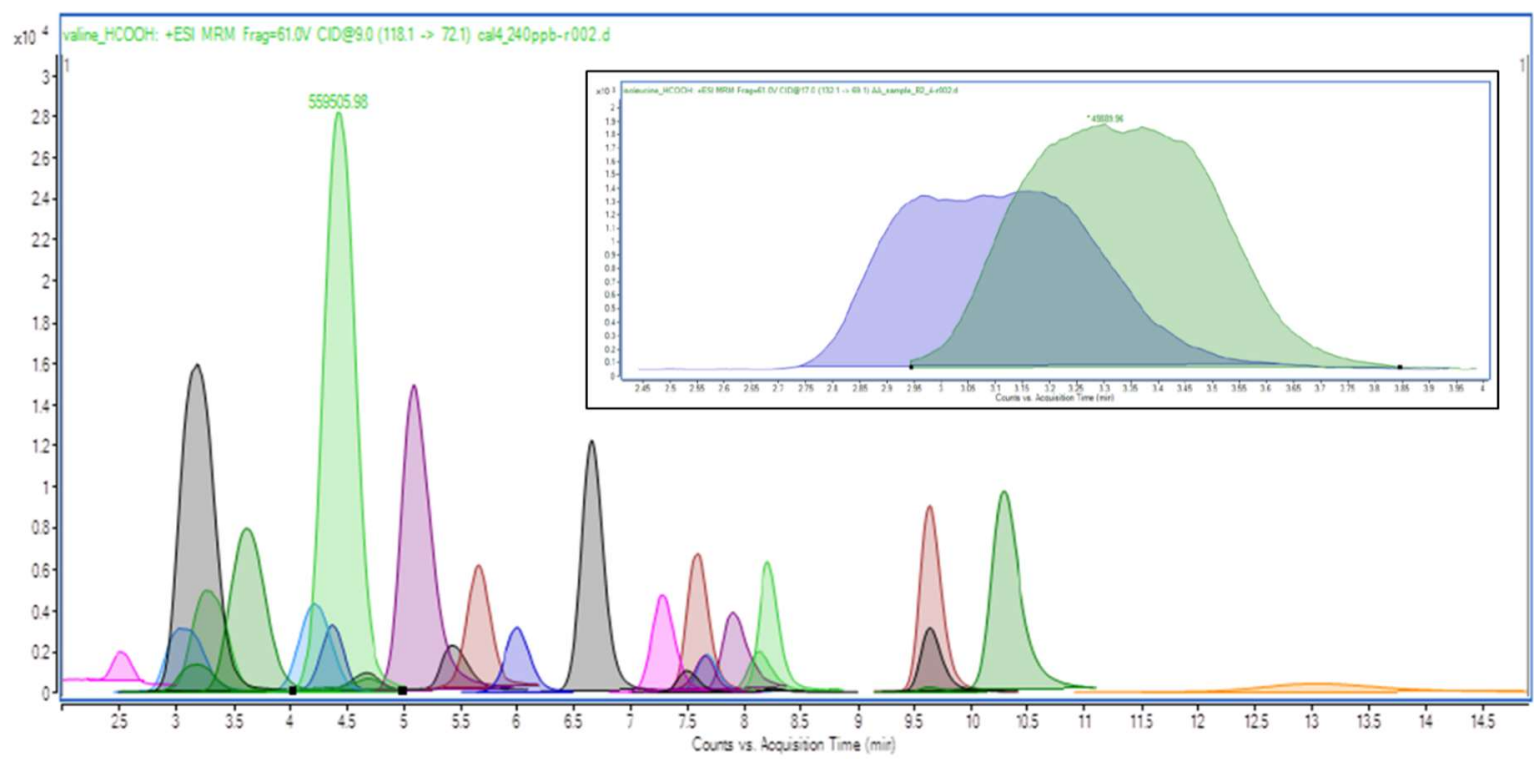

B

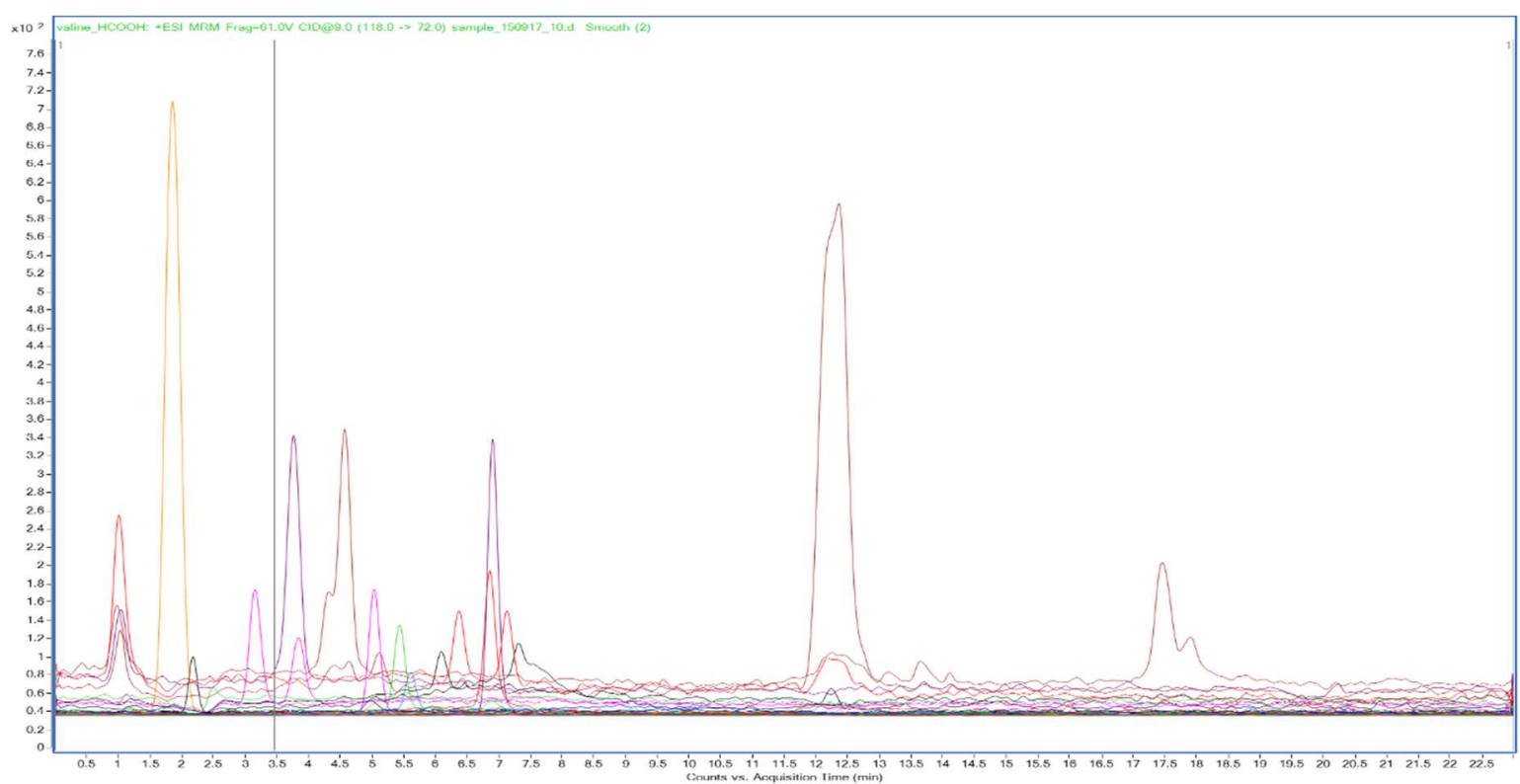

Figure S1. Typical HILIC chromatograms for a standard solution containing $250 \mathrm{ng} \mathrm{mL}^{-1}$ of each analyte (A) and an aerosol sample (B). Detailed separation between Ile and Leu is provided as insert in the chromatogram provided for standard solutions. 


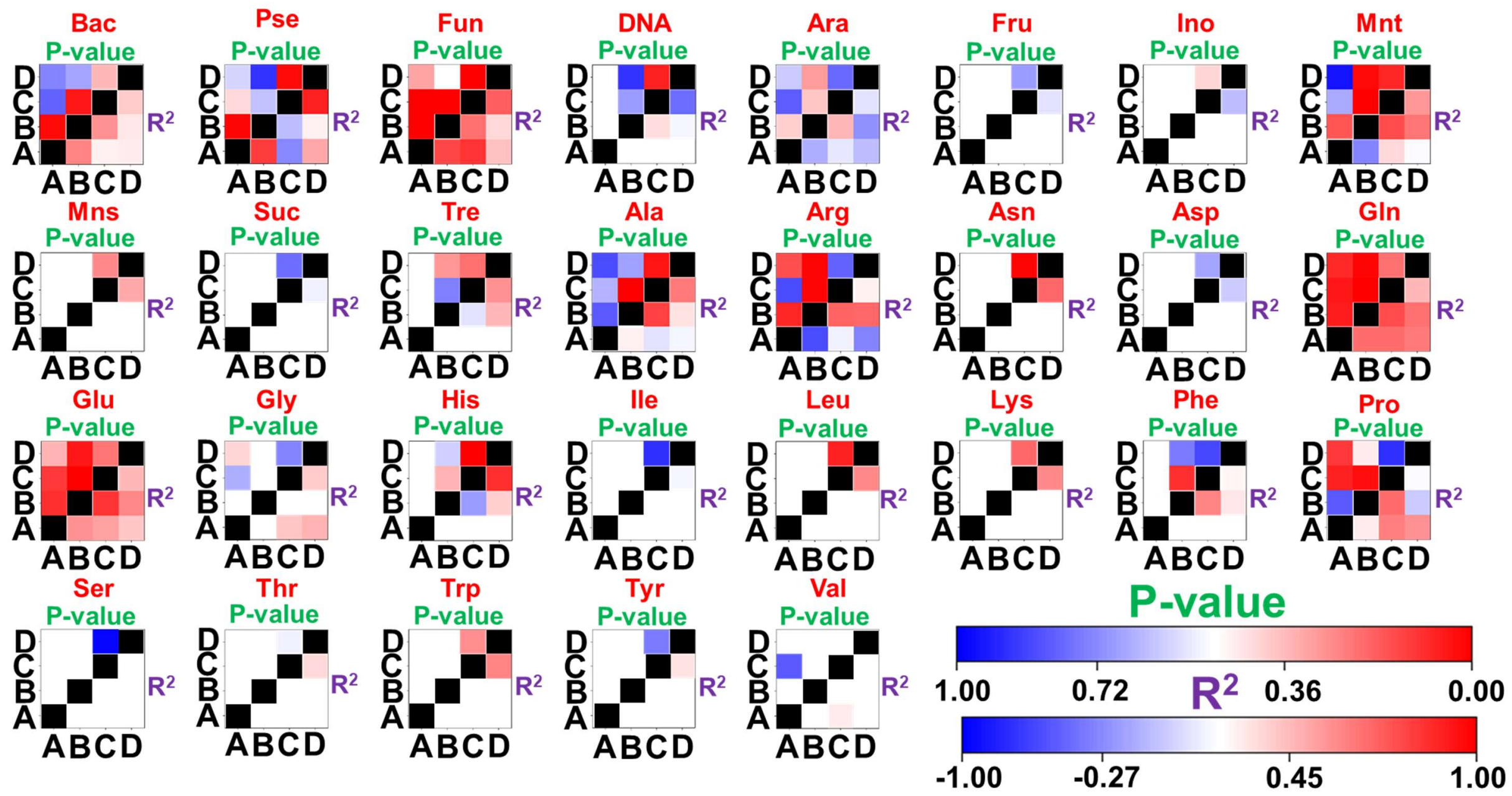

Figure S2. Pearson correlation for the individual microbial groups and chemical compounds detected in PBAPs as a function of the particle size. $\mathrm{P}$ - and R2 values are shown in the upper and lower part of the heat map, respectively. Negative $\mathrm{R}^{2}$ values are used in the figure to show negative correlations. A, $<1.0 \mu \mathrm{m}$ particles $1 ; \mathbf{B}, 1.0-2.5 \mu \mathrm{m}$ particles; $\mathbf{C}, 2.5-10.0 \mu \mathrm{m}$ particles; and $\mathbf{D}, 10>\mu \mathrm{m}$ particles. Bac, bacteria; Pse,

Pseudomonas; and Fun, fungi. 
10000

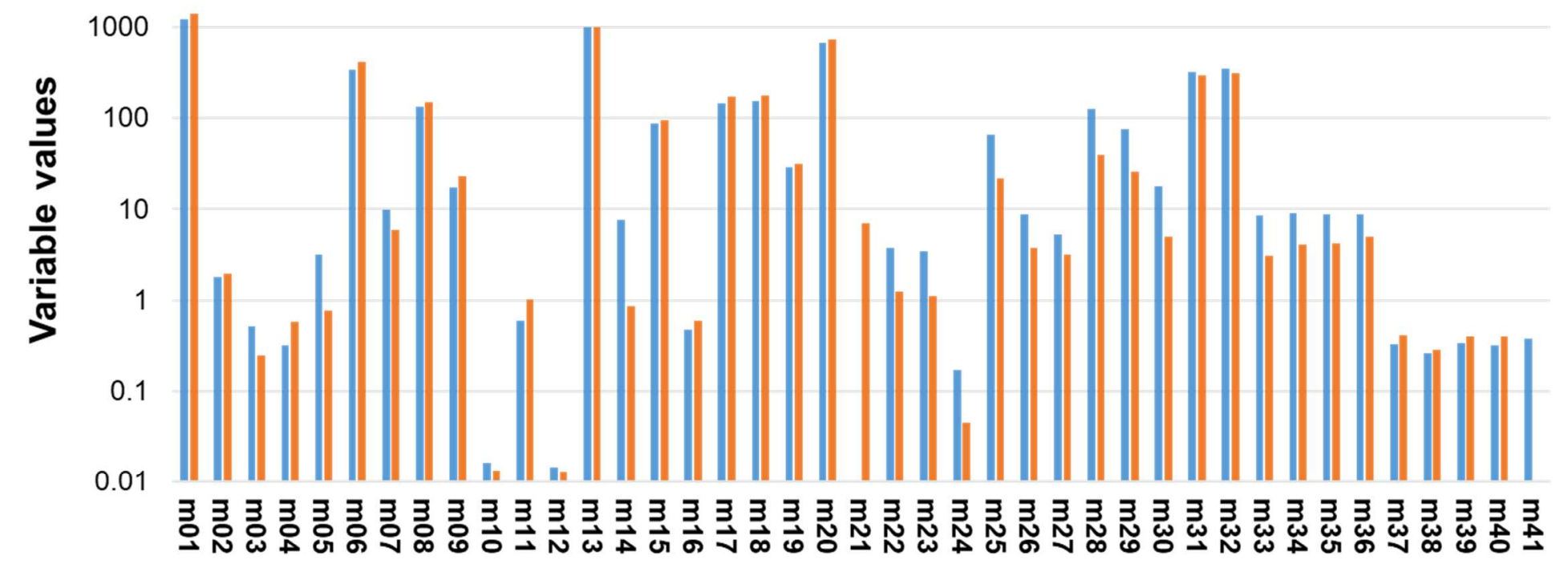

Atmospheric gases concentration, aerosol, meteorological and environmental parameters

Figure S3. Differences between clusters according to the ancillary data. Variable values are shown in logarithmic scale. I Sampling period 1(04.09.2017 to 13.10.2017) and $₫$ Sampling period 2 (23.10.2017 to 22.11.2017). Variable names are coded in Table S4. 


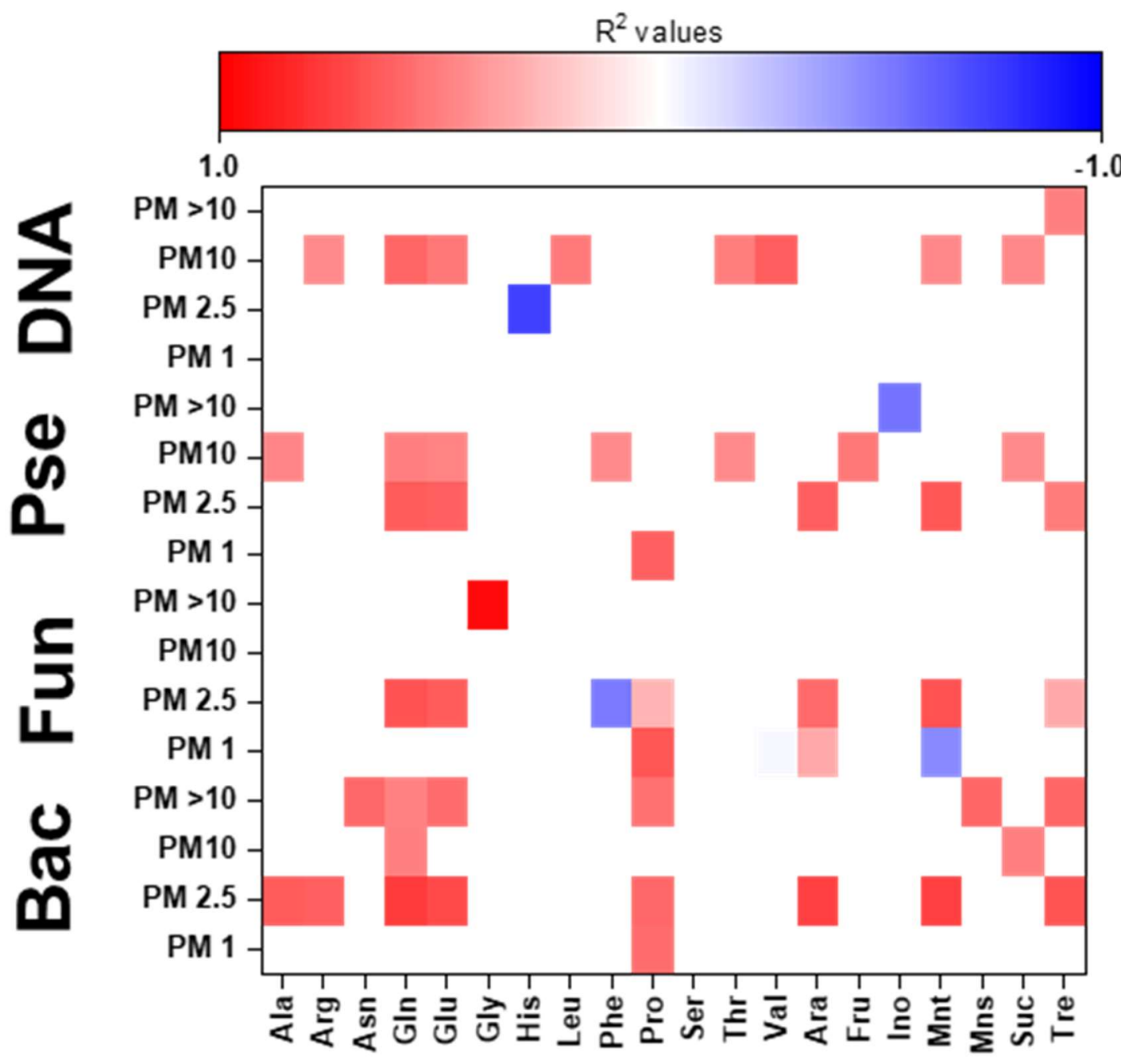

Figure S4. Pearson correlation results between the chemical compounds and the microbial groups, including total DNA, for the different particle sizes. Correlations with P-values $>0.05$ are not shown in this figure. Negative $\mathrm{R}^{2}$ values are used in the figure to show negative correlations between the microbial groups and the chemical species. Bac, bacteria; Pse, Pseudomonas; and Fun, fungi. 


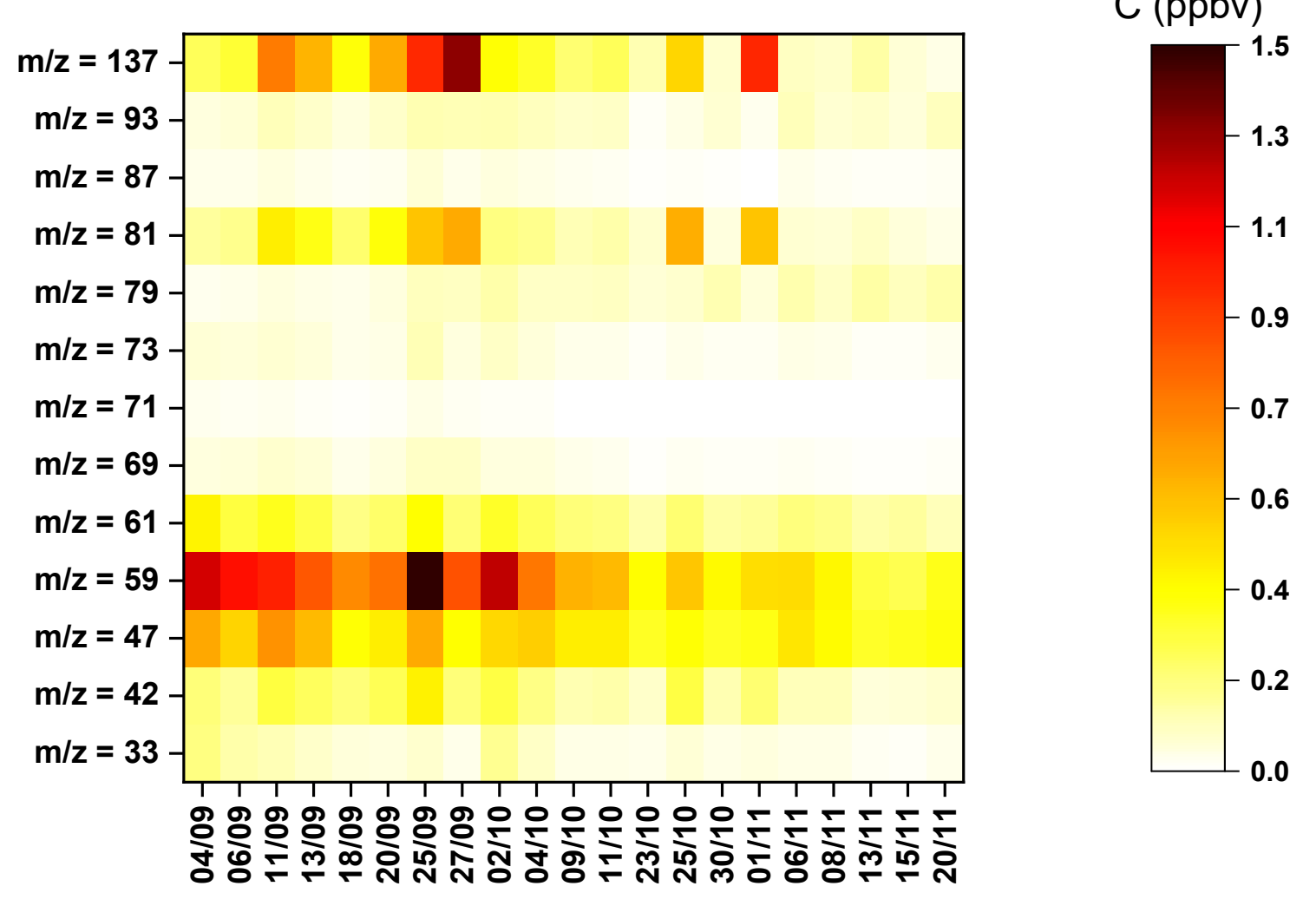

Figure S5. Gas phase VOCs in samples expressed with different $\mathrm{m} / \mathrm{z}$ ions. Initial date of collection is shown in $\mathrm{x}$-axis for the different samples. Concentration is expressed as ppbv. 
Fig. S6 A

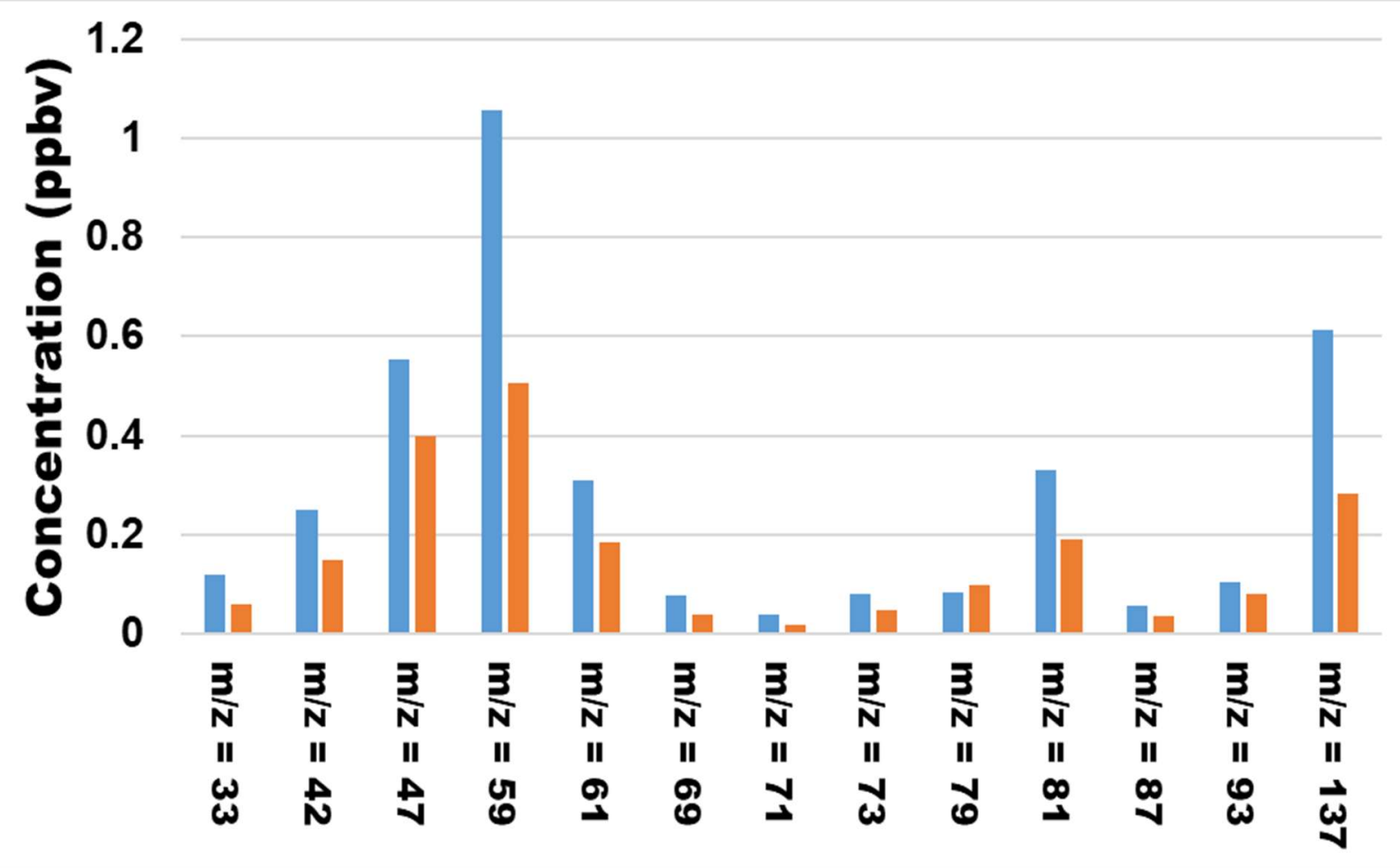


Fig. S6 B

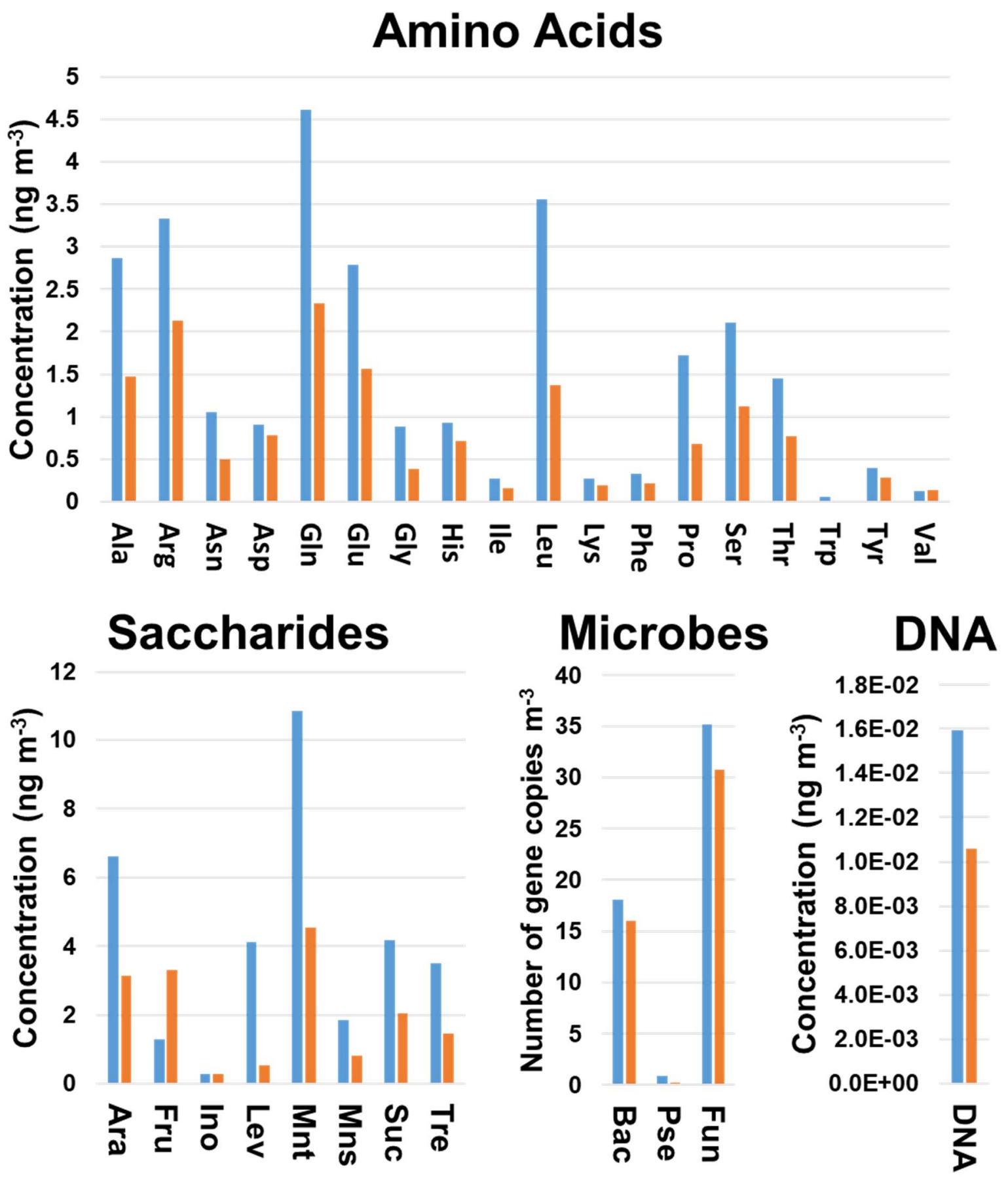

Figure S6. Potential connections between gas phase VOCs and the microbiological composition of PBAPs. A) Differences between clusters based on the gas phase VOCs; B) differences in the chemical and microbiological composition of PBAPs based on the groups established by gas phase VOCs. Average concentrations were calculated for all filter sizes analyzed. @ Group 1 (04.09.2017-15.9.2017 and 25.09.2017-06.10.2017) and $\square$ Group 2 (18.09.2017-22.09.2017 and 09.10.2017-22.11.2017). Bac, bacteria; Pse, Pseudomonas; and Fun, fungi. 


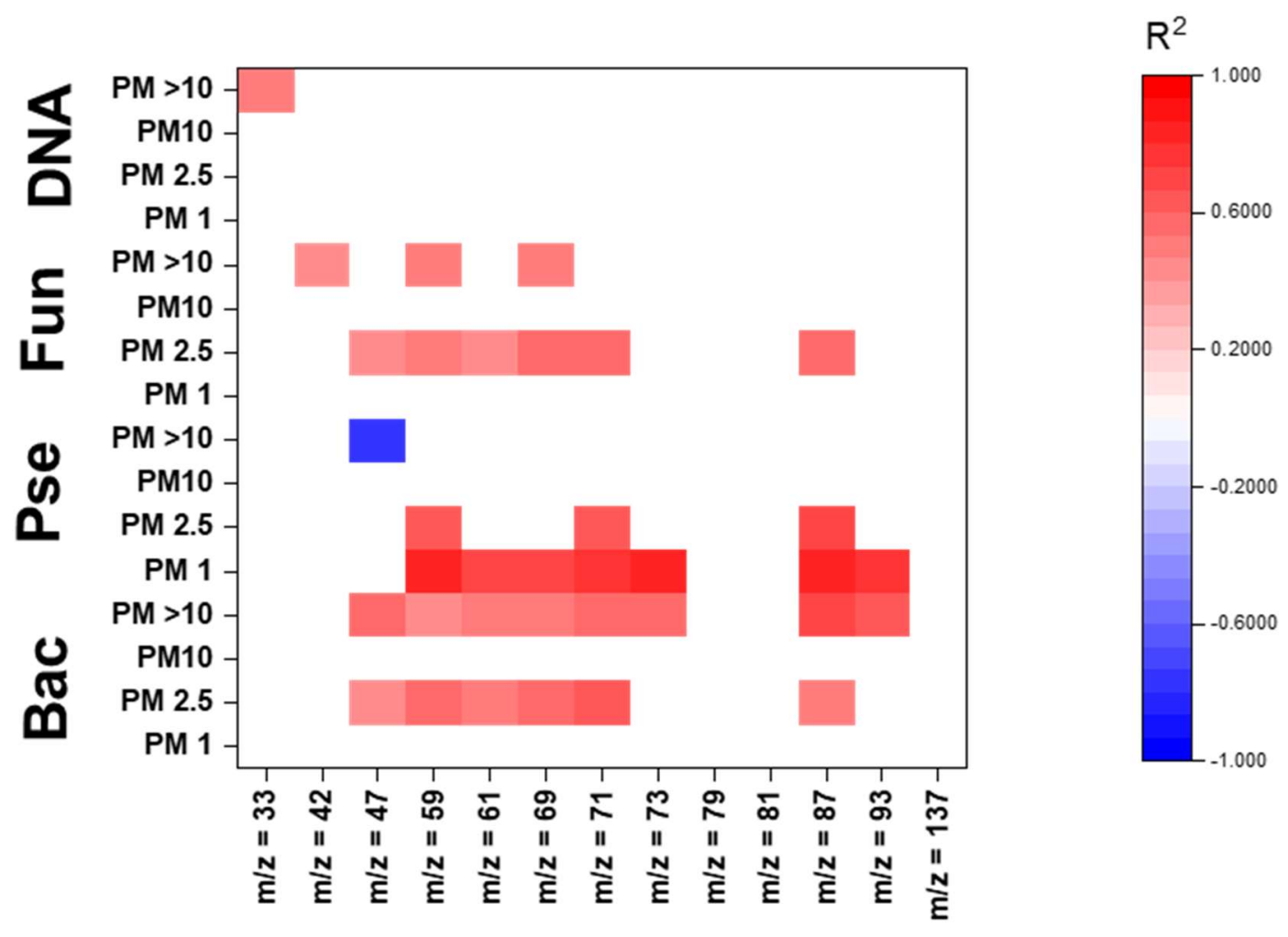

Figure S7. Pearson correlation results for the comparison between the gas phase VOCs and the microbiological composition of PBAPs. Correlations with P-values $>0.05$ are not shown in this figure. Negative R2 values are used in the figure to show negative correlations between the microbial groups and the VOCs. 


\section{References}

de Gouw, J. A., Goldan, P. D., Warneke, C., Kuster, W. C., Roberts, J. M., Marchewka, M., Bertman, S. B., Pszenny, A. A. P., and Keene, W. C.: Validation of proton transfer reaction-mass spectrometry (PTR-MS) measurements of gasphase organic compounds in the atmosphere during the New England Air Quality Study (NEAQS) in 2002, J. Geophys. Res., 108, D21, 4682 https://doi.org/10.1029/2003JD003863, 2003.

de Gouw, J. and Warneke, C.: Measurements of volatile organic compounds in the earth's atmosphere using proton-transferreaction mass spectrometry, Mass Spectrom. Rev., 26, 223-257. https://doi.org/10.1002/mas.20119, 2007.

Rantala, P., Aalto, J., Taipale, R., Ruuskanen, M. T., and Rinne, J.: Annual cycle of volatile organic compound exchange between a boreal pine forest and the atmosphere. Biogeosciences 12, 5753-5770. https://doi.org/10.5194/bg-12-5753$\underline{2015}, 2015$

Taipale, R., Ruuskanen, T. M., Rinne, J., Kajos, M. K., Hakola, H., Pohja, T., and Kulmala, M.: Technical Note: Quantitative long-term measurements of VOC concentrations by PTR-MS - measurement, calibration, and volume mixing ratio calculation methods, Atmos. Chem. Phys., 8, 6681-6698, https://doi.org/10.5194/acp-8-6681-2008, 2008.

Yuan, B., Koss, A. R., Warneke, C., Coggon, M., Sekimoto, K. and de Gouw, J. A.: Proton-Transfer-Reaction Mass Spectrometry: Applications in Atmospheric Sciences, Chem. Rev., 117 (21), 13187-13229, https://doi.org/10.1021/acs.chemrev.7b00325, 2017. 